\title{
LINEAR AND NONLINEAR EXTENSIONS OF LIPSCHITZ FUNCTIONS FROM SUBSETS OF METRIC SPACES
}

\author{
A. BRUDNYI AND Y. BRUDNYI
}

\begin{abstract}
A relationship is established between the linear and nonlinear extension constants for Lipschitz functions defined on subsets of metric spaces. Proofs of several results announced in our earlier paper are presented.
\end{abstract}

\section{$\S 1$. INTRODUCTION}

Let $(M, d)$ be a metric space with the underlying set $M$ and the metric $d$. In what follows we suppress the symbol $d$ in this notation and simply refer to $M$ as a metric space. Let $B$ be a Banach space with norm $\|\cdot\|$. The space $\operatorname{Lip}(M, B)$ of Banach-valued Lipschitz functions $f: M \rightarrow B$ is determined by the seminorm

$$
|f|_{\operatorname{Lip}(M, B)}:=\sup _{m \neq m^{\prime}} \frac{\left\|f(m)-f\left(m^{\prime}\right)\right\|}{d\left(m, m^{\prime}\right)} .
$$

In case $B=\mathbb{R}$, we denote the corresponding space by $\operatorname{Lip}(M)$. Regarding a subset $S \subset M$ as the metric space equipped with the induced metric, we introduce the family of spaces $\operatorname{Lip}(S, B)$.

Definition 1.1. A subset $S \subset M$ admits a simultaneous Lipschitz extension if there exists a linear bounded operator $T: \operatorname{Lip}(S) \rightarrow \operatorname{Lip}(M)$ such that

$$
\left.T f\right|_{S}=f, \quad f \in \operatorname{Lip}(S) .
$$

The set of all such operators is denoted by $\operatorname{Ext}(S, M)$, and the optimal extension constant is given by

$$
\lambda(S, M):=\inf \{\|T\|: T \in \operatorname{Ext}(S, M)\} .
$$

(We assume that $\lambda(S, M)=\infty$ if $\operatorname{Ext}(S, M)=\emptyset$.) Finally, we define the global linear Lipschitz extension constant:

$$
\lambda(M):=\sup \{\lambda(S, M): S \subset M\} .
$$

We say that $M$ satisfies the linear Lipschitz extension property if $\lambda(M)<\infty$.

In the same way we define the nonlinear Lipschitz extension constant $\nu(S, M, B)$ to be the infimum of the constants $C$ such that every $f \in \operatorname{Lip}(S, B)$ admits an extension to a function $\tilde{f} \in \operatorname{Lip}(M, B)$ satisfying $|\widetilde{f}|_{\operatorname{Lip}(M, B)} \leq C|f|_{\operatorname{Lip}(S, B)}$. Then we define

$$
\nu(M, B):=\sup \{\nu(S, M, B): S \subset M\} .
$$

We are ready to present the main result of the paper. For its formulation we let $\mathcal{B}_{\text {fin }}$ denote the category of all finite-dimensional Banach spaces. Also, we set

$$
\nu(M):=\sup \left\{\nu(M, B): B \in \mathcal{B}_{\text {fin }}\right\} .
$$

2000 Mathematics Subject Classification. Primary 54E40.

Key words and phrases. Lipschitz function, linear extension operator, Banach space.

Supported in part by NSERC. 
Theorem 1.2. The following identity is true:

$$
\lambda(M)=\nu(M) .
$$

Let us formulate several consequences of this result.

A convex subset $K$ of a Banach space $B$ containing $0 \in B$ is said to be generating if the union of all its dilations $\lambda K, \lambda \geq 1$, is dense in $B$.

Corollary 1.3. $\lambda(K)<\infty$ for a generating set $K \subset B$ if and only if $\operatorname{dim} B<\infty$.

In particular, $\lambda(B)=\infty$ if $\operatorname{dim} B=\infty$. This naturally leads to the following problem.

Problem 1.4. Find the asymptotics for $\lambda\left(l_{p}^{n}\right)$ as $n \rightarrow \infty$.

We recall that, for any finite-dimensional Banach space $B$, we have

$$
\lambda(B) \leq c \operatorname{dim} B,
$$

where $c$ is a numerical constant bounded by about 4000 in JLSch and by 24 in BB2. The method of the latter paper also gives the following estimates for $p=1, \infty$ and some numerical constant $c>1 / 4$ :

$$
c \sqrt{n} \leq \lambda\left(l_{p}^{n}\right) \leq 24 n, \quad n \in \mathbb{N} .
$$

As a consequence of our Theorem 1.2 and [LN, Lemma 1.13] we shall obtain a lower estimate for the case where $p=2$. Together with Theorem 1.11 of the above cited paper, this leads to the following statement.

Corollary 1.5. For some numerical constants $0<c_{1}<c_{2}$ we have

$$
c_{1} n^{1 / 8} \leq \lambda\left(l_{2}^{n}\right) \leq c_{2} n^{1 / 2} .
$$

In Theorem 2.18 of BB2 we constructed a metric graph $G$ of bounded geometry such that $\operatorname{Ext}(S, G)=\emptyset$ for some subspace $S \subset G$; in particular, $\lambda(G)=\infty$. Our next result makes it possible to construct one-dimensional metric spaces with the latter property that "almost coincide" with the real line (i.e., $\mathbb{R}$ equipped with the standard metric). To formulate the result we recall several definitions.

A map $f:\left(M_{0}, d_{0}\right) \rightarrow\left(M_{1}, d_{1}\right)$ is called a $C$-isometric embedding, $C>0$, if

$$
C^{-1} d_{0}\left(m^{\prime}, m^{\prime \prime}\right) \leq d_{1}\left(f\left(m^{\prime}\right), f\left(m^{\prime \prime}\right)\right) \leq C d_{0}\left(m^{\prime}, m^{\prime \prime}\right)
$$

for all $m^{\prime}, m^{\prime \prime} \in M_{0}$. We also call such an $f$ a quasi-isometric embedding if the constant $C$ is inessential.

Next, if for every $m \in M_{0}$ there is an open ball $B \subset M_{0}$ centered at $m$ such that the above inequality is fulfilled for some $C=C(B)>0$ and all $m^{\prime}, m^{\prime \prime} \in B$, then the map $f$ is called a locally quasi-isometric embedding.

Finally, a map $f:\left(M_{0}, d_{0}\right) \rightarrow\left(M_{1}, d_{1}\right)$ is called an $L$-rough similarity, $L>0$, if

$$
d_{0}\left(m^{\prime}, m^{\prime \prime}\right)-L \leq d_{1}\left(f\left(m^{\prime}\right), f\left(m^{\prime \prime}\right)\right) \leq d_{0}\left(m^{\prime}, m^{\prime \prime}\right)+L
$$

for all $m^{\prime}, m^{\prime \prime} \in M_{0}$.

Corollary 1.6. Let $(M, d)$ be a complete separable length space 1 Then for every $\epsilon>0$ there is a metric space $\left(M_{\epsilon}, d_{\epsilon}\right)$ with the following properties:

(a) $\lambda(M) \leq \lambda\left(M_{\epsilon}\right)$;

(b) $M_{\epsilon}$ is homeomorphic and locally quasi-isometric to the real line. Moreover, there is a closed subset $F \subset \mathbb{R}$ of measure $\epsilon$ such that $\mathbb{R} \backslash F$ admits an isometric embedding in $M_{\epsilon}$. If, in addition, diam $M<\infty$, then $M_{\epsilon}$ is $\epsilon$-roughly similar to the real line;

\footnotetext{
${ }^{1}$ I.e., $d\left(m, m^{\prime}\right)=\inf _{\gamma} l(\gamma)$, where the infimum is taken over all curves in $M$ joining $m$ and $m^{\prime}$, and $l(\gamma)$ stands for the length of $\gamma$.
} 
(c) $M_{\epsilon}$ is a proper metric space (i.e., every closed ball in $M_{\epsilon}$ is compact).

Though $\operatorname{dim} M_{\epsilon}=1$, this space is metrically as large as the space $M$ (which may be of dimension infinity). In a sense, $M_{\epsilon}$ may be viewed as a kind of Peano type curve. The following assertion confirms this claim.

Corollary 1.7. Assume that $M_{\epsilon}$ admits a quasi-isometric embedding into a proper metric space $\widetilde{M}$ with a cocompact action of the group of isometries 2 Then $M$ also admits a quasi-isometric embedding in $\widetilde{M}$.

In particular, $M_{\epsilon}$ cannot be embedded in any such space if $M$ is a closed ball of an infinite-dimensional Banach space. Also, in this case there is a subspace $S \subset M_{\epsilon}$ such that $\operatorname{Ext}\left(S, M_{\epsilon}\right)=\emptyset$.

\section{§2. Finiteness PROPERTY}

In the proof of Theorem 1.2 we use some results on the linear Lipschitz extension constants, established in BB2]; we formulate these results in a form suitable for our consideration.

Definition 2.1. Let $\left\{\left(M_{i}, d_{i}\right)\right\}_{i \in \mathbb{N}}$ be a sequence of metric spaces. This sequence is said to be $\gamma$-convergent to a metric space $(M, d)$ if for every compact subspace $K$ of $M$ there is a sequence $\left\{K_{i} \subset M_{i}: i \in I \subset \mathbb{N}\right\}$ of compact subspaces that converges to $K$ in the Gromov-Hausdorff metric.

For the reader's convenience, we recall the definition of the Gromov-Hausdorff distance $d_{\mathcal{G H}}\left(M_{0}, M_{1}\right)$ between metric spaces $\left(M_{i}, d_{i}\right), i=0,1$ (see, e.g., [BH], pp. 70-75] for more details).

Let $d$ be a metric on the disjoint union $M_{0} \sqcup M_{1}$ whose restriction to $M_{i}$ coincides with $d_{i}, i=0,1$. Then each $M_{i}$ is a subspace of the space $\left(M_{0} \sqcup M_{1}, d\right)$, and the Hausdorff distance

$$
d_{\mathcal{H}}\left(M_{0}, M_{1}\right):=\sup \left\{\max _{i=0,1} d\left(m_{i}, M_{1-i}\right): m_{i} \in M_{i}\right\}
$$

is well defined. Then the Gromov-Hausdorff metric is given by

$$
d_{\mathcal{G H}}\left(M_{0}, M_{1}\right):=\inf _{d} d_{\mathcal{H}}\left(M_{0}, M_{1}\right)
$$

where $d$ runs over all such metrics on $M_{0} \sqcup M_{1}$.

Our first formulation is based on [BB2, Theorem 2.2 and Remark 6.1].

Theorem A. (a) Let $\left\{\left(M_{i}, d_{i}\right)\right\}_{i \in \mathbb{N}}$ be a sequence of metric spaces $\gamma$-convergent to a metric space $(M, d)$. Then

$$
\lambda(M) \leq \limsup _{i \rightarrow \infty} \lambda\left(M_{i}\right) .
$$

(b) Let $S$ be a subspace of $(M, d)$. Then

$$
\lambda(S, M)=\sup _{F} \lambda(F, M),
$$

where $F$ runs over all finite subsets of $S$.

Now, let $S$ be a subset of $M$ satisfying the following property.

For some bi-Lipschitz map $\delta: M \rightarrow M$,

(A) $S \subset \delta(S)$;

(B) $\bigcup_{j=0}^{\infty} \delta^{j}(S)$ is dense in $M$;

\footnotetext{
${ }^{2}$ This means that for some compact set $K \subset \widetilde{M}$ and every point $m \in \widetilde{M}$ there is an isometry $I$ of $\widetilde{M}$ such that $I(m) \in K$.
} 
(C) for the linear operator $\Delta: \operatorname{Lip}(M) \rightarrow \operatorname{Lip}(M)$ given by $(\Delta f)(m):=f(\delta(m))$, $m \in M$, we have

$$
\|\Delta\| \cdot\left\|\Delta^{-1}\right\|=1
$$

Then the following is true (see Corollary 2.5 in $[\mathrm{BB} 2$ ).

Theorem B. If $S \subset M$ satisfies conditions $(\mathrm{A})-(\mathrm{C})$, then

$$
\lambda(S)=\lambda(M) .
$$

\section{§3. Auxiliary Results}

The proof of Theorem 1.2 is based on several auxiliary results from [BB2, §5]; see also [K, §3]. We present them below, referring to the cited papers for the proofs. In their formulations, we use the notion of a pointed metric space, that is, a metric space $M$ with a distinguished point $m_{0}$. A (pointed) metric subspace of such a space is a subset of $M$ containing $m_{0}$ and endowed with the induced metric. We denote by $\operatorname{Lip}_{0}(M, B)$ the subspace of $\operatorname{Lip}(M, B)$ distinguished by the condition $f\left(m_{0}\right)=0, f \in \operatorname{Lip}(M, B)$. The notation $\operatorname{Lip}_{0}(S, B)$ for a metric subspace $S$ of $M$ has a similar meaning. Note that all these spaces are Banach.

Now, let $K(M)$ be the canonical predual space to $\operatorname{Lip}_{0}(M)$ (the KantorovichRubinshtein space). Recall that $K(M)$ is the closed linear span of the set of the point evaluations

$$
\delta_{m}(f):=f(m), \quad f \in \operatorname{Lip}_{0}(M),
$$

in the dual space $\operatorname{Lip}_{0}(M)^{*}$ (see, e.g., [BB2, Appendix]).

Lemma 3.1. Let $\left(M, d, m_{0}\right)$ be a pointed metric space. Then:

(1) there is an isometric embedding $i_{M}$ of $M$ in $\operatorname{Lip}_{0}(M)^{*}$ such that $i_{M}\left(m_{0}\right)=0$;

(2) the map $m \mapsto \delta_{m}$ is an isometric embedding of $M$ in $K(M)$.

This lemma allows us to regard $M$ as a metric subspace of either $\operatorname{Lip}_{0}(M)^{*}$ or $K(M)$.

Lemma 3.2. Suppose $E \in \operatorname{Ext}(S, M)$ and $\operatorname{card} S<\infty$. There exists a linear bounded operator $E_{0}: K(M) \rightarrow K(S)$ such that

(1) $E_{0}$ is predual to $E$; in particular, $\left\|E_{0}\right\|=\|E\|$;

(2) $E_{0}$ is a projection onto $K(S)$.

Lemma 3.3. If $f \in \operatorname{Lip}_{0}(M, B)$, then there is a linear bounded operator $T_{f}: K(M) \rightarrow B$ such that

(1) $\left.T_{f}\right|_{M}=f$ (here $M$ is identified with its isometric copy in $K(M)$ );

(2) $\left\|T_{f}\right\|=|f|_{\operatorname{Lip}(M, B)}$.

\section{$\S 4$. Proofs}

Proof of Theorem 1.2, First, we prove

Claim I.

$$
\nu(M) \leq \lambda(M) .
$$

The proof is based on the following two inequalities, the first of which asserts that

$$
\nu(F, M, B) \leq \lambda(F, M)
$$

provided $\operatorname{card} F<\infty$.

To formulate the second inequality, we define a "finite-dimensional" analog of the constant $\nu(S, M, B)$ by the formula

$$
\nu_{\text {fin }}(S, M, B):=\sup \{\nu(F, M, B): F \subset S, \operatorname{card} F<\infty\} .
$$


Then, for $\operatorname{dim} B<\infty$, we have

$$
\nu(S, M, B) \leq \nu_{\text {fin }}(S, M, B)
$$

We shall prove these inequalities later; now we derive Claim I from them.

In (4.1), we choose a Banach space $B$ of class $\mathcal{B}_{\text {fin }}$ and take the supremum over all $F \subset S$ with card $F<\infty$. Using (4.2), we get

$$
\nu_{\text {fin }}(S, M, B) \leq \sup \{\lambda(F, M): F \subset S, \operatorname{card} F<\infty\} .
$$

By Theorem A, the right-hand side equals $\lambda(S, M)$. Together with (4.3), this leads to the inequality

$$
\nu(S, M, B) \leq \lambda(S, M) .
$$

Taking the supremum over all $B \in \mathcal{B}_{\text {fin }}$ and $S \subset M$, we prove Claim I.

Now we establish inequalities (4.1) and (4.3). In their proofs, $M$ is a pointed metric space with a distinguished point $m_{0}$, and all subsets of $M$ are assumed to be pointed subspaces of $M$ (with the distinguished point $m_{0}$ ).

To prove (4.1), pick $F \subset M$ with card $F<\infty$. For $f \in \operatorname{Lip}_{0}(F, B)$, let $T_{f}$ be the linear operator of Lemma 3.3. Hence, $T_{f}: K(F) \rightarrow B$ and

$$
\left\|T_{f}\right\|=L(f):=|f|_{\operatorname{Lip}(F, B)} .
$$

Given an extension operator $E \in \operatorname{Ext}(F, M)$, we find the corresponding predual operator $E_{0}: K(M) \rightarrow K(F)$ of Lemma 3.2 and put

$$
E_{f}:=T_{f} E_{0} .
$$

Then $E_{f}: K(M) \rightarrow B$, and the norm of $E_{f}$ is at most $\left\|E_{0}\right\| \cdot\left\|T_{f}\right\|=L(f)\|E\|$. By Lemma 3.3. $\left.E_{f}\right|_{M}: M \rightarrow B$ is a Lipschitz extension of $f$ from $F$ to the whole of $M$. Moreover,

$$
L\left(\left.E_{f}\right|_{M}\right) \leq\left\|E_{f}\right\| \leq L(f)\|E\| .
$$

By the definition of the nonlinear Lipschitz extension constant, this implies

$$
\nu(F, M, B) \leq\|E\| .
$$

Passing to the infimum over all $E \in \operatorname{Ext}(F, M)$, we get (4.1).

To prove (4.3), for a given $\epsilon>0$ we find a function $f \in \operatorname{Lip}_{0}(S, B)$ with $L(f)=1$ that admits an $\epsilon$-optimal extension to $M$; this means that there exists $\widetilde{f} \in \operatorname{Lip}_{0}(M, B)$ such that

$$
-2 \epsilon+\nu(S, M, B) \leq-\epsilon+L(\widetilde{f}) \leq \inf \left\{L(g): g \in \operatorname{Lip}_{0}(M, B),\left.g\right|_{S}=f\right\} .
$$

Let $\mathcal{F}$ be the family of all finite subsets of $S$, partially ordered by inclusion. For every $F \in \mathcal{F}$, we define $g_{F}$ to be an $\epsilon$-optimal extension of the trace $\left.f\right|_{F}$ to $M$, that is,

$$
-\epsilon+L\left(g_{F}\right) \leq \inf \left\{L(g): g \in \operatorname{Lip}_{0}(M, B),\left.g\right|_{F}=\left.f\right|_{F}\right\} .
$$

By the definition of $\tilde{f}$, for all $F \in \mathcal{F}$ we get

$$
L\left(g_{F}\right) \leq L(\widetilde{f})+\epsilon .
$$

Hence, the family $\left\{g_{F}\right\}_{F \in \mathcal{F}}$ is equicontinuous. It is also uniformly bounded on every ball in $M$, because $g_{F}\left(m_{0}\right)=f\left(m_{0}\right)=0$ for all $F$. Finally, every $g_{F}$ maps $M$ into the finitedimensional Banach space $B$. Therefore, a standard compactness argument involving the Tychonov theorem (see, e.g., [BB2, §5]), allows us to find a subnet of the net $\left\{g_{F}\right\}_{F \in \mathcal{F}}$ that converges pointwise to a Lipschitz function $g: M \rightarrow B$. By the definition of net convergence, we have

$$
\left.g\right|_{S}=f
$$


Next, passing to the limit in (4.5) and using (4.2), we also obtain

$$
\begin{aligned}
-\epsilon+L(g) & \leq-\epsilon+\sup _{F \in \mathcal{F}} L\left(g_{F}\right) \\
& \leq \sup _{F \in \mathcal{F}} \inf \left\{L(g): g \in \operatorname{Lip}_{0}(M, B),\left.g\right|_{F}=\left.f\right|_{F}\right\} \leq \nu_{\text {fin }}(S, M, B) .
\end{aligned}
$$

Since $\widetilde{f}$ is an $\epsilon$-optimal extension of $f$ (see (4.4) ), from (4.6) we see that

$$
L(\widetilde{f}) \leq L(g)+\epsilon .
$$

Together with the preceding inequality and (4.4), this yields

$$
\nu(S, M, B) \leq L(\widetilde{f})+\epsilon \leq \nu_{\mathrm{fin}}(S, M, B)+3 \epsilon .
$$

This completes the proofs of (4.3) and Claim I.

Claim II.

$$
\lambda(M) \leq \nu(M) .
$$

We derive this fact from the inequality

$$
\lambda(F, M) \leq \nu(F, M):=\sup \left\{\nu(F, M, B): B \in \mathcal{B}_{\text {fin }}\right\},
$$

valid for $\operatorname{card} F<\infty$.

Taking here the supremum over all such $F$ and applying Theorem A with $S=M$, we get

$$
\lambda(M)=\sup _{F} \lambda(F, M) \leq \sup _{F} \nu(F, M) \leq \nu(M),
$$

as required.

It remains to establish (4.7).

Let $\delta: F \rightarrow K(F)$ be the isometric embedding described in Lemma 3.1. Then $\delta \in \operatorname{Lip}(F, K(F))$, and by the definition of $\nu(F, M)$ for a given $\epsilon>0$ there is an extension $\widetilde{\delta} \in \operatorname{Lip}(M, K(F))$ such that

$$
-\epsilon+L(\widetilde{\delta}) \leq \nu(F, M) L(\delta)(=\nu(F, M)) .
$$

Let $f \in \operatorname{Lip}_{0}(F)$. Since $\operatorname{Lip}_{0}(F)=K(F)^{*}$, the function $f$ can be regarded as a linear bounded functional on $K(F)$. We define a linear operator $E: \operatorname{Lip}_{0}(F) \rightarrow \operatorname{Lip}_{0}(M)$ by

$$
(E f)(m):=\langle f, \widetilde{\delta}(m)\rangle_{K(F)} \text {. }
$$

Then the norm $\|E f\|_{\operatorname{Lip}_{0}(M)}$ is bounded by

$$
\|f\|_{\operatorname{Lip}_{0}(F)} \sup _{m \neq m^{\prime}} \frac{\left\|\widetilde{\delta}(m)-\widetilde{\delta}\left(m^{\prime}\right)\right\|_{K(F)}}{d\left(m, m^{\prime}\right)}=L(f) L(\widetilde{\delta}) \leq(\nu(F, M)+\epsilon) L(f) .
$$

Hence,

$$
\|E\| \leq \nu(F, M)+\epsilon
$$

and moreover,

$$
\left.(E f)\right|_{F}=f,
$$

i.e., $E \in \operatorname{Ext}(F, M)$. By the definition of $\lambda(F, M)$, this implies inequality (4.7):

$$
\lambda(F, M) \leq \nu(F, M) .
$$

Finally, from the inequalities of Claims I and II we get the required statement of Theorem 1.2 . 
Proof of Corollary 1.5. The following fact was established in the proof of Lemma 1.13 of the paper $[\mathrm{LN}$; see Remark 5.3 there.

Let $2<p<\infty$ and $m \in \mathbb{N}$. There is a finite subset $F \subset l_{2}^{2 m}$ with card $F \leq m^{2 m}$ and a numerical constant $c>0$ such that

$$
\nu\left(F, l_{2}^{2 m}, l_{p}^{2 m}\right) \geq c\left(\frac{2 m \log 2 m}{\log (2 m \log 2 m)}\right)^{\frac{p-2}{p^{2}}} .
$$

Choosing $p=4$, we get

$$
\nu\left(F, l_{2}^{2 m}, l_{4}^{2 m}\right) \geq c m^{1 / 8}
$$

The definition implies that

$$
\sup \left\{\nu\left(l_{2}^{2 m}, B\right): B \in \mathcal{B}_{\text {fin }}\right\} \geq \nu\left(l_{2}^{2 m}, l_{4}^{2 m}\right) \geq \sup \nu\left(\Gamma, l_{2}^{2 m}, l_{4}^{2 m}\right),
$$

where $\Gamma$ runs over all finite point metric subspaces of $l_{2}^{2 m}$. These two inequalities show that

$$
\sup \left\{\nu\left(l_{2}^{2 m}, B\right): B \in \mathcal{B}_{\text {fin }}\right\} \geq c m^{1 / 8} .
$$

Finally, by Theorem 1.2, the left-hand side equals $\lambda\left(l_{2}^{2 m}\right)$, and this yields the required lower bound

$$
\lambda\left(l_{2}^{n}\right) \geq c_{1} n^{1 / 8}, \quad n \in \mathbb{N}
$$

with some constant $c_{1}>0$ independent of $n$. The upper bound

$$
\lambda\left(l_{2}^{n}\right) \leq c_{2} n^{1 / 2}, \quad n \in \mathbb{N},
$$

was in fact proved in $[\mathrm{LN}]$, since these authors used a linear extension operator to prove the estimate

$$
\nu\left(l_{2}^{n}, B\right) \leq c n^{1 / 2}, \quad n \in \mathbb{N}
$$

(see Theorem 1.11 and part 5 of Theorem 5.1 in $[\mathrm{LN}]$ ).

Proof of Corollary 1.3. Take $\delta(x):=2 x, x \in B$, and let $S$ be the generating set $K$ of the corollary. We check that the conditions (A) $-(\mathrm{C})$ of Theorem B are fulfilled. Since $K$ is convex and contains 0 , we have

$$
K \subset \delta(K):=2 K
$$

For the same reason, $\bigcup_{i=1}^{\infty} \delta^{i}(K)=\bigcup_{\lambda \geq 1} \lambda K$, and the latter set is dense in $B$ by the definition of a generating set. Finally, the operator $\Delta: \operatorname{Lip}(B) \rightarrow \operatorname{Lip}(B)$ is now defined by $(\Delta f)(x):=f(2 x), x \in B$, so that $\|\Delta\|=2$ and $\left\|\Delta^{-1}\right\|=1 / 2$. Hence, Theorem B yields

$$
\lambda(K)=\lambda(B) .
$$

Let, in particular, $U_{B}$ be the closed unit ball of the Banach space $B$. Clearly, this is a generating set; therefore, (4.8) implies

$$
\lambda\left(U_{B}\right)=\lambda(B) .
$$

Applying this to the Banach space $l_{2}^{n}$ and using the lower bound mentioned in Corollary 1.5. we get

$$
\lambda\left(U_{l_{2}^{n}}\right) \geq c_{1} n^{1 / 8}, \quad n \in \mathbb{N} .
$$

Now, let $B$ be an infinite-dimensional Banach space. By the Dvoretzky theorem $[\mathrm{D}$, for every sufficiently large $n$, say $n \geq n_{0}$, there is an $n$-dimensional linear subspace $B_{n} \subset B$ such that the ball $U_{B} \cap B_{n}$ is 2-isometric to $U_{l_{2}^{n}}$. In other words, there is a bi-Lipschitz map $\phi$ of $U_{l_{2}^{n}}$ onto $U_{B} \cap B_{n}$ such that

$$
\max \left\{L(\phi), L\left(\phi^{-1}\right)\right\} \leq 2 .
$$


This immediately implies the inequality

$$
\lambda\left(U_{B}\right) \geq \lambda\left(U_{B} \cap B_{n}\right) \geq \frac{1}{4} \lambda\left(U_{l_{2}^{n}}\right) .
$$

Together with (4.10) and (4.9), this shows that, for $n \geq n_{0}$,

$$
\lambda(B)=\lambda\left(U_{B}\right) \geq \frac{1}{4} c_{1} n^{1 / 8} \rightarrow \infty \text { as } n \rightarrow \infty .
$$

Consequently,

$$
\lambda(B)=\infty \quad \text { if } \operatorname{dim} B=\infty .
$$

In the case where $n:=\operatorname{dim} B<\infty$, the classical Whitney theorem W] implies that $\lambda(B) \leq c(n)<\infty$; see also the above-cited inequality [JLSch].

Proof of Corollary 1.6. Given a complete separable length metric space $(M, d)$, we choose a monotone increasing sequence of finite subspaces $M_{n} \subset M, n \in \mathbb{N}$, such that card $M_{n}=$ $n, \bigcup_{n \in \mathbb{N}} M_{n}$ is dense in $M$ and, moreover, all $M_{n}$ contain a distinguished point $m^{*} \in M$.

Let $m_{1}, \ldots, m_{n}$ be points of $M_{n}$ enumerated in some way so that $m_{1}=m^{*}$. We also set $m_{n+1}:=m^{*}$. We join $m_{i}$ and $m_{i+1}$ by a curve $\gamma_{i}$ of length $l\left(\gamma_{i}\right) \leq 2 d\left(m_{i}, m_{i+1}\right)$, $1 \leq i \leq n$. Let $f_{n}$ denote the concatenation (union) of all $\gamma_{i}, 1 \leq i \leq n$. Then $f_{n}$ is a closed curve in $M$ with the endpoint $m^{*}$, having length $\sum_{i=1}^{n} l\left(\gamma_{i}\right)$, and passing through all points of $M_{n}$. The reparametrization of $f_{n}$ by arclength divided by an appropriate positive number turns this map into a Lipschitz map $g_{n}:\left[n-1, n-1+\frac{\epsilon}{2^{n}}\right] \rightarrow$ image $f_{n} \subset$ $M$ passing through all the points of $M_{n}$ and such that

$$
g_{n}(n-1)=g_{n}\left(n-1+\epsilon / 2^{n}\right)=m^{*} .
$$

Now, let $(\widehat{M}, \widehat{d})$ be the metric space with the underlying set $M \times \mathbb{R}$ and the metric $\widehat{d}$ given by the formula $\widehat{d}\left((m, t),\left(m^{\prime}, t^{\prime}\right)\right):=\max \left\{d\left(m, m^{\prime}\right),\left|t-t^{\prime}\right|\right\}$. We denote the graph of $g_{n}$ by $h_{n}:=\left\{\left(g_{n}(t), t\right): n-1 \leq t \leq n-1+\frac{\epsilon}{2^{n}}\right\} \subset \widehat{M}$. Using the family $\left\{h_{n}\right\}_{n \in \mathbb{N}}$, we define a locally Lipschitz embedding $h: \mathbb{R} \rightarrow \widehat{M}$ by

$$
h(t):= \begin{cases}\left(m^{*}, t\right) & \text { if } t \leq 0, \\ h_{n}(t) & \text { if } t \in J_{n}^{-}, \quad n \in \mathbb{N}, \\ \left(m^{*}, t\right) & \text { if } t \in J_{n}^{+}, \quad n \in \mathbb{N} .\end{cases}
$$

Here $J_{n}^{-}:=\left[n-1, n-1+\frac{\epsilon}{2^{n}}\right]$ and $J_{n}^{+}:=\left[n-1+\frac{\epsilon}{2^{n}}, n\right]$. By (4.11), this determines a homeomorphism of $\mathbb{R}$ onto the metric subspace $M_{\epsilon}:=h(\mathbb{R})$ of the space $\widehat{M}$. Clearly, $M_{\epsilon}$ is also locally quasi-isometric to $\mathbb{R}$ (equipped with the standard metric). Moreover, in accordance with the definition, the set $\mathbb{R} \backslash\left(\bigcup_{n \in \mathbb{N}} J_{n}^{-}\right)$equipped with the standard metric is isometric under $h$ to a subspace of $M_{\epsilon}$. If, in addition, diam $M<\infty$, then we replace the metric $d$ on $M$ by the equivalent metric $\widetilde{d}:=\epsilon d /(2 \operatorname{diam} M)$ and then construct the metric space $M_{\epsilon}$ as above for the space $(M, \widetilde{d})$. Clearly, this space is $\epsilon$-roughly similar to $\mathbb{R}$.

This completes the proof of assertion (b) of the corollary.

Now we prove assertion (a). Let $T_{n}$ be an $n$-pointed subset of the interval $J_{n}^{-}$such that $h\left(T_{n}\right)=M_{n} \times T_{n}$ and $n-1 \in T_{n}$. Then

$$
\bigcup_{n \in \mathbb{N}} M_{n} \times T_{n} \subset M_{\epsilon} .
$$

We translate $M_{n} \times T_{n}$ along $\mathbb{R}$ by $1-n$, and denote the resulting set by $S_{n}$. Then $S_{n} \subset M_{n} \times\left[0, \frac{\epsilon}{2^{n}}\right] \subset \widehat{M}$. We shall show that the sequence $\left\{S_{n}\right\}_{n \in \mathbb{N}} \gamma$-converges to 
$M \times\{0\} \subset \widehat{M}$. Then, since $S_{n}$ is isometric to $M_{n} \times T_{n}$, Theorem A and (4.12) yield the desired inequality:

$$
\lambda(M)=\lambda(M \times\{0\}) \leq \limsup _{n \rightarrow \infty} \lambda\left(M_{n} \times T_{n}\right) \leq \lambda\left(M_{\epsilon}\right) .
$$

To establish the $\gamma$-convergence of $\left\{S_{n}\right\}_{n \in \mathbb{N}}$, consider an arbitrary compact set $\widehat{K} \subset$ $M \times\{0\}$; then $\widehat{K}=K \times\{0\}$ for some compact set $K$ of $M$. Let $K_{n}$ denote the intersection of the $\epsilon / 2^{n}$-neighbourhood of $K$ in $M$ with $M_{n}$, and let $\widehat{K}_{n}:=\left(K_{n} \times\left[0, \frac{\epsilon}{2^{n}}\right]\right) \cap S_{n}$. Since $\bigcup_{n \in \mathbb{N}} M_{n}$ is dense in $M$ and the sequence $\left\{M_{n}\right\}_{n \in \mathbb{N}}$ is monotone increasing, $\widehat{K}_{n} \neq \emptyset$ for sufficiently large $n$. Then, for such an $n$, the Hausdorff distance from $\widehat{K}$ to $\widehat{K}_{n}$ is at most $\frac{\epsilon}{2^{n}}$. Hence, the compact sets $\widehat{K}_{n} \subset S_{n}$ with sufficiently large $n$ form a sequence convergent in the Hausdorff metric to $\widehat{K}$. By Definition 2.1. this means that $\left\{S_{n}\right\}_{n \in \mathbb{N}}$ $\gamma$-converges to $M \times\{0\}$.

Thus, assertion (a) is also proved.

It remains to check that the space $M_{\epsilon}$ is proper (assertion (c)). Let $\bar{B}_{r}(m) \subset M_{\epsilon}$ be the closed ball of $M_{\epsilon}$ centered at $m$ and of radius $r$. Let $p_{\mathbb{R}}: \widehat{M} \rightarrow \mathbb{R}$ denote the canonical projection onto the second component of $\widehat{M}=M \times \mathbb{R}$. By our definition of $M_{\epsilon}$, this ball is contained in the image $h\left(\left[p_{\mathbb{R}}(m)-r, p_{\mathbb{R}}(m)+r\right]\right)$ of the compact interval $\left[p_{\mathbb{R}}(m)-r, p_{\mathbb{R}}(m)+r\right]$ of $\mathbb{R}$. Since $h$ is continuous, this image is compact. Therefore, the closed subset $\bar{B}_{r}(m)$ of this image is also compact.

This proves assertion (c).

Proof of Corollary 1.7. Let $f: M_{\epsilon} \rightarrow \widetilde{M}$ be a $C$-isometric embedding in a proper metric space $\widetilde{M}$ with the cocompact action of its group of isometries. Let $\left\{S_{n}\right\}_{n \in \mathbb{N}} \subset \widetilde{M}$ be the sequence defined above. By the construction of $S_{n}$, the map $f$ determines a $C$ isometric embedding $f_{n}: S_{n} \rightarrow \widetilde{M}$. We choose an isometry $I_{n}$ of $\widetilde{M}$ such that, for some fixed compact set $K \subset \widetilde{M}$, the point $\left(m^{*}, 0\right) \in S_{n}$ goes to a point of $K$ under the map $\widehat{f}_{n}:=I_{n} \circ f_{n}$. For $k \geq n$, let $S_{n k}$ denote a subset of $S_{k}$ whose canonical projection onto the space $M$ coincides with $M_{n}$. Since $M_{n} \subset M_{k}$ for $k \geq n$, such a set exists and is unique. Since the point $\left(m^{*}, 0\right)$ belongs to every $S_{n k}$ with $k \geq n$, the point $\widehat{f}_{n}\left(m^{*}, 0\right)$ belongs to $K$. Therefore, $\widehat{f}_{n}\left(S_{n k}\right)$ lies in the $\lambda$-neighbourhood of $K$ with $\lambda:=C \max \left\{\operatorname{diam} M_{n}, \frac{\epsilon}{2^{n}}\right\}$. Since the space $\widetilde{M}$ is proper, the closure of this neighbourhood, denoted by $K_{n}$, is compact. Now we identify $S_{n k}$ for $k \geq n$ with $M_{n}$ by the canonical projection $p_{M}: \widehat{M} \rightarrow M$ and denote by $\phi_{n k}: M_{n} \rightarrow K_{n}$ the map $\widehat{f_{k}} \circ p_{M}^{-1}$, where $p_{M}^{-1}$ is the map inverse to $\left.p_{M}\right|_{S_{n k}}$. Since the sequence of finite sets $\left\{S_{n k}\right\}_{k \geq n}$ converges to $M_{n} \times\{0\}$ in the Hausdorff metric of the space $\widehat{M}$, the map $\phi_{n k}$ is a $C_{k}$-isometric embedding and $C_{k}$ tends to $C$ as $k \rightarrow \infty$. Also, we clearly have

$$
\left.\phi_{m k}\right|_{M_{n}}=\phi_{n k} \quad \text { for all } k \geq m \geq n \text {. }
$$

Since the target space of each $\phi_{n k}$ is the compact set $K_{n}$, we can use (4.13) and the Cantor diagonal process to find a subsequence $\left\{\phi_{n k(n)}\right\}_{n \in \mathbb{N}}$ that converges pointwise on the dense subset $\bigcup_{n \in \mathbb{N}} M_{n}$ of $M$, and its limit, say $\widetilde{f}$, is a $C$-isometric embedding in $\widetilde{M}$. Extending $\widetilde{f}$ to the entire $M$ by continuity, we obtain the desired quasi-isometric embedding of $M$ in $\widetilde{M}$.

The remaining statements of the corollary follow straightforwardly from its first part and [BB2, Theorem 2.8]. 


\section{REFERENCES}

[BB1] A. Brudnyı̆ and Yu. Brudnyı̆, Simultaneous extensions of Lipschitz functions, Uspekhi Mat. Nauk 60 (2005), no. 6, 53-72; English transl., Russian Math. Surveys 60 (2005), no. 6, $1057-$ 1076. MR2215754 (2007f:26004)

[BB2] Metric spaces with linear extensions preserving Lipschitz condition, Amer. J. Math. 129 (2007), 217-314. MR2288741

[BH] M. Bridson and A. Haefliger, Metric spaces of non-positive curvature, Grundlehren Math. Wiss., Bd. 319, Springer-Verlag, Berlin, 1999. MR1744486 (2000k:53038)

[D] A. Dvoretzky, Some results on convex bodies and Banach spaces, Proc. Internat. Sympos. Linear Spaces (Hebrew Univ., Jerusalem, 1960), Jerusalem Acad. Press, Jerusalem, 1961, pp. 123-160. MR0139079 (25:2518)

[JLSch] W. B. Johnson, J. Lindenstrauss, and G. Schechtman, Extensions of Lipschitz maps into Banach spaces, Israel J. Math. 54 (1986), no. 2, 129-138. MR0852474 (87k:54021)

[K] N. Kalton, Spaces of Lipschitz and Hölder functions and their applications, Collect. Math. $\mathbf{5 5}$ (2004), no. 2, 171-217. MR.2068975 (2005c:46113)

[LN] J. R. Lee and A. Naor, Extending Lipschitz functions via random metric partitions, Invent. Math. 160 (2005), no. 1, 59-95. MR2129708(2006c:54013)

[W] H. Whitney, Analytic extensions of differentiable functions defined in closed sets, Trans. Amer. Math. Soc. 36 (1934), 63-89. MR 1501735

Department of Mathematics and Statistics, University of Calgary, Calgary, Canada

E-mail address: albru@math.ucalgary.ca

Department of Mathematics, Technion, Haifa, Israel

E-mail address: ybrudnyi@math.technion.ac.il

Received 20/APR/2006

Originally published in English 